\title{
An empirical study on the influence of on-road static obstacles on driver behaviour
}

\author{
Bhupali Dutta ${ }^{1}$ and Vinod Vasudevan ${ }^{2, *}$ \\ ${ }^{1}$ Department of Civil Engineering, Indian Institute of Technology, Kanpur 208 016, India \\ ${ }^{2}$ University of Alaska, Anchorage, 2900 Spirit Drive, AK 99508, USA
}

Understanding the influence of static obstacles on driver behaviour is important because drivers' instinctual reactions may pose serious hazards to safety and mobility. The effects of road geometry, roadside infrastructure and static objects near the road on driver behaviour have been studied extensively. However, no studies discuss the influence of two variables that are to likely to affect driver behaviour: obstacle size (width and length) and the side on which the static obstacle is present (driver's side or passenger's side). Controlled experiments were conducted using an instrumented vehicle to collect instantaneous driver behaviour data in the presence of on-road static obstacles. The results show that the side on which the obstacle is present influences the lateral gap. The results also show that obstacle size, both in terms of width and length, affects driving speed and the lateral gap. The results of this study can aid in the analysis of safety and mobility issues related to partial lane closures, stopped vehicles and similar obstacles.

Keywords: Driver behaviour, obstacle size, on-road static obstacles, lateral gap, vehicle speed.

VEHICLES frequently stop on roads for a variety of reasons, including mechanical failure, flat tires or police intervention. If shoulders are present, vehicles can pull over without obstructing the roadway. However, in many instances, vehicles stop on road segments without shoulders, resulting in full or partial blockage of the carriageway. Similarly, there are often lane closures following a vehicle crash that may block the carriageway. Since these events are unplanned, there is no way to provide sufficient advanced warning to drivers. Obstacles present on the carriageway influence drivers significantly, especially when they are introduced without advanced warning. The behaviour of a driver in the presence of an obstacle on the carriageway also has cascading effects on upstream traffic and can lead to serious consequences, such as dangerous driving or traffic bottlenecks. Understanding drivers' actions in the presence of various obstacles is therefore important for both safety and mobility considerations.

*For correspondence. (e-mail: vvasudevan@alaska.edu)
The visual field is the fundamental display for drivers. The drivers gather most information visually. Different objects subtend different visual angles based on their size. Perceived risk depends on the size of the obstacle; the larger the obstacle, higher is the anticipated risk ${ }^{1,2}$. Once drivers detect an object, they determine its hazard potential. Whether an object is sizeable enough to constitute a hazard depends on the road width and relative size of the object with other familiar objects ${ }^{3}$. Additionally, the side of the static obstacle can influence the lateral gap maintained from the obstacle. When the obstacle is present on a vehicle's near side (the driver's side), the drivers can clearly see the obstacle and estimate its lateral gap. On the other hand, when the obstacle is present on the far side (the passenger's side), the drivers may not be able to estimate the lateral gap accurately. However, no studies exist that discuss either the influence of different dimensions of obstacle size or the side on which the static obstacle is present.

The objective of the present study is to understand the influence of static obstacles on speed and lateral gap, with the side on which the obstacle is present and two dimensions of obstacle size (width and length) serving as the key independent variables.

\section{Literature review}

Driver behaviour is influenced by various factors such as driver experience, and attitude as well as road environment. The different elements of road environment include geometric and infrastructure elements, lane closures, static objects near the road, etc.

\section{Influence of road geometric elements (road width and shoulder width)}

Researchers have studied the influence of narrow roads and road width reduction on speed profiles and lateral placement $^{4-6}$. These studies reported that road width reductions resulted in a significant reduction in vehicle speed. On narrow roads, drivers prefer to drive closer to the road's centre and avoid overtaking other drivers. Green et $a l^{7}$ observed that the standard deviation in 


\section{RESEARCH ARTICLES}

lateral position increased when the roads became wider as drivers tend to become more relaxed.

Some studies have reported that narrow roads reduce driving speed and thus increase road safety ${ }^{8-10}$, whereas wide roads provide drivers with the opportunity to accelerate to dangerous speeds ${ }^{11-13}$ and thus increase the likelihood and magnitude of accidents.

\section{Influence of static objects near the road edge}

Studies have been conducted to examine whether different types of objects (parked vehicles, barricades), located at different distances from the road edge on multilane highways, have an effect on lateral position, reduction in speed and lateral gap from other vehicles ${ }^{14,15}$. It was observed that narrower the road and closer the object (for example, equilateral triangle in the study by Michaels and Cozan ${ }^{14}$, and car, truck and barricade in the study by Taragin ${ }^{15}$ ) to the road edge, greater was the displacement of the vehicles from their normal paths. Vehicle speed was little affected by the presence of roadside objects on wide roads and in low to moderate traffic conditions. There was a diminishing influence of the roadside object as the number of lanes on the road increased. The type of object did not have a significant influence on the amount of lateral displacement. In a driving simulator study, Van der Horst and de Ridder ${ }^{16}$ observed that the type of safety barrier had no impact on travel speed and lateral displacement; only its presence affected the driver.

\section{Influence of specific features of static objects on driver behaviour}

Much research has been conducted on the presence and location of static obstacles on safety and driving performance. However, there has only been limited research on the influence of certain specific features of the object, such as its type, orientation and direction of appearance (static and dynamic). Michaels and Cozan ${ }^{14}$ analysed different factors of static obstacles on lateral displacement and vehicle speed. One of the factors was orientation of the obstacle. In the first orientation, the base of an equilateral triangle was closer to the pavement, while in the second orientation the apex of the triangle was closer to it. Results from the study indicated that the lateral displacement of the vehicle was higher when the apex of the triangle was closer to the pavement. The difference increased with increase in travel speed and decreased as the object distance increased from the travel path. Participant's age and direction of appearance of obstacle (front or periphery) also influenced the minimum braking distance towards the obstacle. In a simulator study by Martin et $a l .{ }^{17}$ the authors found that older drivers maintained larger lateral gaps than younger drivers. In their study, the obstacle was on-road pedestrians. The minimum distance was larger when the pedestrian appeared from the periphery compared to the front. However, minimum distance was not significantly different when the pedestrian appeared from the left or the right.

Understanding the influence of object size on the perceived likelihood of collision is important for the analysis of safety and design of interception mechanisms. Several studies have been conducted to understand the effect of size-arrival of objects on collision avoidance and interception mechanisms ${ }^{1,18,19}$. The studies found that participants viewed larger object to be closer and anticipated that a larger object would arrive first, even if it was farther away. Similar observations were recorded when different sizes of objects had the same heights and were approached from the same distance at the same speed. During the experiments, both width and length of the objects were varied. Caird and Hancock ${ }^{20}$ studied participants' reactions in a traffic scenario in which different sizes of vehicles approached a left turn. They found that participants perceived that larger vehicles could reach the left turn sooner than smaller vehicles. Similarly, Levulis et $a .^{21}$ used a driving simulator to examine whether overtaking judgements depend on the size of the oncoming vehicle. The results showed that more accepted gaps and false alarms occurred when the oncoming vehicle was a motorcycle than when it was a car or truck.

\section{Objectives}

Although there are several studies on the influence of the presence, type and location of static objects in the road environment, there are no studies on the influence side of presence of static obstacle and influence of different dimensions of size of the obstacle. Hence, in the present study we examine the following: (i) the influence of side of the static obstacle on gap-to-width ratio. (ii) The influence of width of the static obstacle on driving speed and gap-to-width ratio. (iii) The influence of length of the static obstacle on driving speed and gap-to-width ratio.

\section{Experiment}

The method of data collection, experimental design and participants are discussed below.

\section{Method of data collection}

Data were collected using an instrumented vehicle, which allows for detailed and accurate collection of instantaneous driver and vehicle response data. The vehicle was equipped with VLP-16 Lidar, four video cameras, IMU-GPS and 

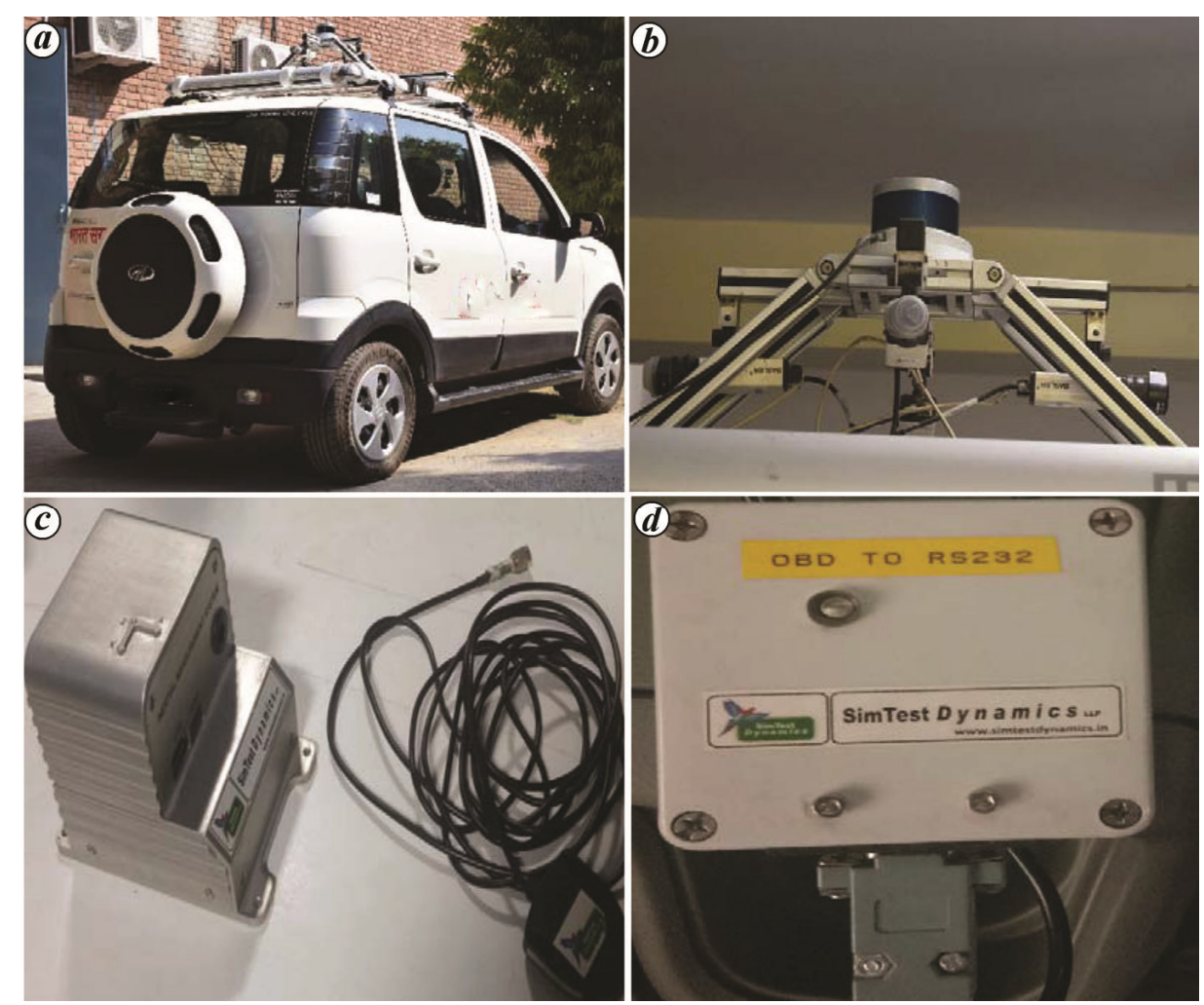

Figure 1. $\boldsymbol{a}$, Instrumented vehicle. $\boldsymbol{b}$, Lidar and video cameras mounted on the roof of the vehicle. $\boldsymbol{c}$, IMU-GPS unit. $\boldsymbol{d}$, OBD scanner.

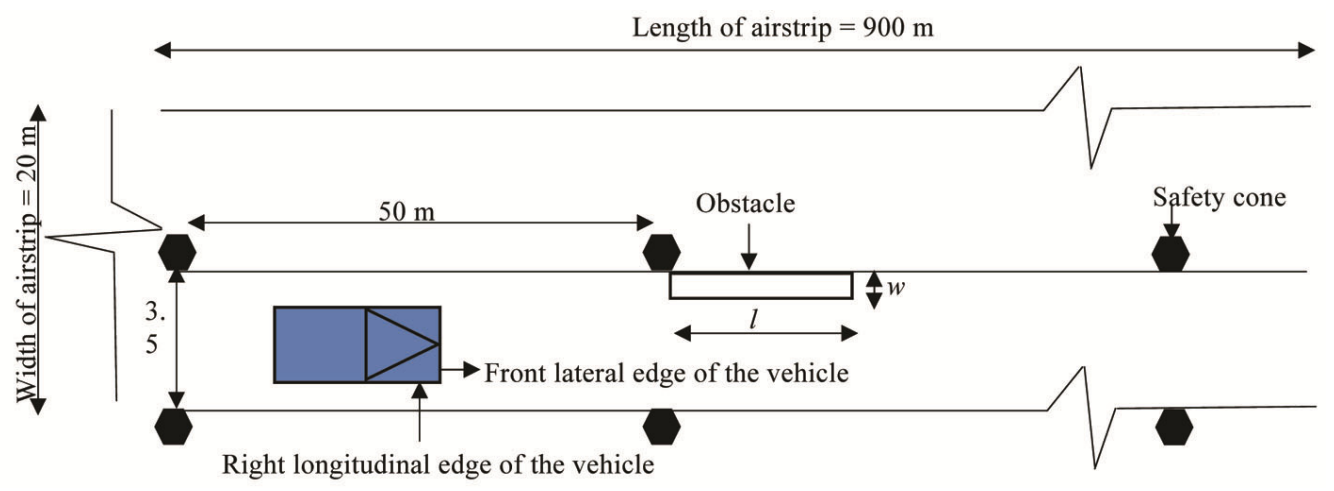

Figure 2. Schematic of test track.

on-board diagnostics (OBD) scanner. The position coordinates $(x, y, z)$ of various objects in the surrounding can be obtained with the help of Lidar; the position of the vehicle and its lateral acceleration can be obtained from the IMU-GPS unit, and speed of the vehicle can be obtained from the OBD scanner. Data collection was done by recording with the help of video cameras. To track objects detected by the Lidar, an object tracking and data extraction algorithm was written in Matlab. The rest of the information (instrumented vehicle position, lateral acceleration and speed) were recorded in .csv files.

The instrumented vehicle was a sports utility vehicle (SUV), $3.98 \mathrm{~m}$ long and $1.85 \mathrm{~m}$ wide. The Lidar and video cameras were mounted on the roof of the vehicle. Figure $1 a-c$ provides a snapshot of the vehicle, location of the video cameras and Lidar, OBD scanner and IMU-GPS unit. The sampling frequency of the sensors was $10 \mathrm{~Hz}$.

\section{Experimental design}

Drivers are influenced by various factors, which makes it difficult to interpret driver behaviour in specific cases. To avoid uncontrollable external factors, the experiments were conducted on a test track, an airstrip that is $900 \mathrm{~m}$ 


\section{RESEARCH ARTICLES}

long and $20 \mathrm{~m}$ wide. A $3.5 \mathrm{~m}$ wide track was developed with traffic safety cones, which were placed at an interval of $50 \mathrm{~m}$ along the length of the track. Two static obstacles were placed using traffic safety cones to reduce the track width in two sections by 0.5 and $1 \mathrm{~m}$. The track width was reduced for lengths of 25 and $100 \mathrm{~m}$, making four static objects of different dimensions. To study the influence of the side of the static object, these four objects were first placed on the driver's side and then on the passenger's side. Figures 2 and 3 show the schematic diagram and snapshot of the prepared test track for the experiment respectively.

From previously conducted trial experiments, it was observed that at least $100 \mathrm{~m}$ of length from the start of the test track is required to attain the desired speed. Hence, the first obstacle was introduced after a distance of $150 \mathrm{~m}$ from the start point.

\section{Participants}

Five male drivers, with a minimum of five years of driving experience, participated in the experiment. The participants had no prior knowledge of the purpose of the experiment. Besides the driver, an analyst was also present in the vehicle during data collection. The drivers were instructed to drive within the $3.5 \mathrm{~m}$ wide track at speeds of $30 \mathrm{~km} / \mathrm{h}(8.4 \mathrm{~m} / \mathrm{s}), 40 \mathrm{~km} / \mathrm{h}(11.2 \mathrm{~m} / \mathrm{s})$ and $50 \mathrm{~km} / \mathrm{h}(14 \mathrm{~m} / \mathrm{s})$, as instructed by the analyst. No further instructions were given to the drivers and the analyst had no further interactions with them.

\section{Definition of variables}

Drivers perceive obstacles as a risk. The magnitude of risk may depend on the size of the obstacle, driving speed

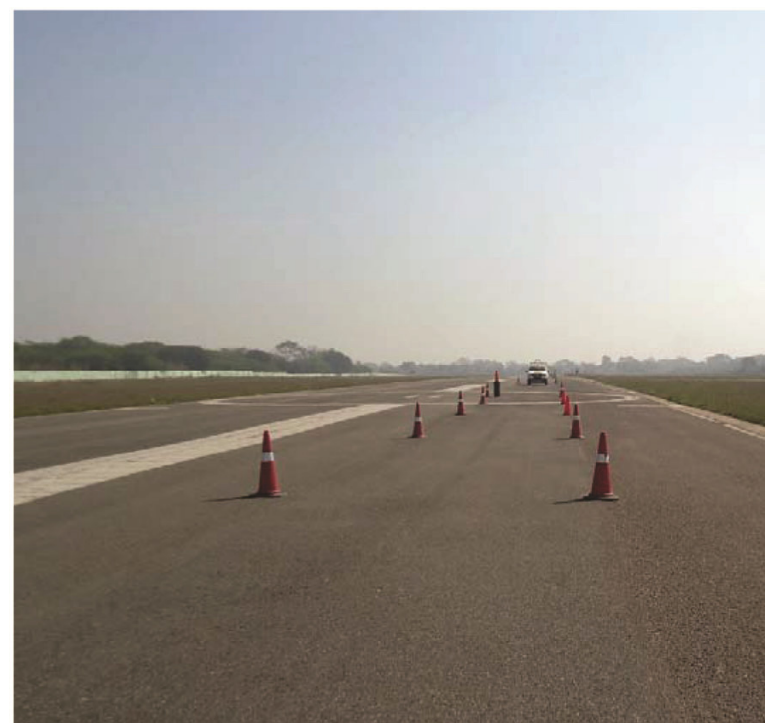

Figure 3. Snapshot of test track. and duration of exposure. As the size of the obstacle, driving speed and duration of exposure increase, the perceived risk may also increase. Based on the hazard potential of the obstacle, a driver undertakes suitable countermeasures such as lateral displacement or speed reduction. It is expected that drivers choose a speed and lateral position corresponding to their risk perception.

The side of the vehicle on which the driver's seat is located is referred to as the driver's side and the other side (laterally) is referred to as the passenger's side. In India, where this study was conducted, the driver's side is on the right-hand side of the vehicle (RHS) when facing forward.

In the present study, track width was reduced from 3.5 to $3 \mathrm{~m}$ and 3.5 to $2.5 \mathrm{~m}$ for obstacles of 0.5 and $1 \mathrm{~m}$ width respectively. The hazard potential of an object depends on the road width and relative size of the object with other familiar objects ${ }^{3}$. Hence, in order to normalize the lateral gap for different track widths in the presence of different obstacle widths, the gap-to-width ratio, $g_{\mathrm{w}}(t)$, is calculated. In addition, the deviation in instantaneous speed from the instructed speed, $\Delta V(t)$, is calculated.

Box 1 provides a list of the variables used in this study.

\section{Analysis and results}

Analyses were done to achieve the objectives of this study.

\section{Influence of static obstacle's location on gap-to-width ratio}

When an obstacle is on the driver's side, the driver can clearly perceive the lateral gap. However, when the obstacle is on the passenger's side, the driver may not be able to estimate the lateral gap accurately since the

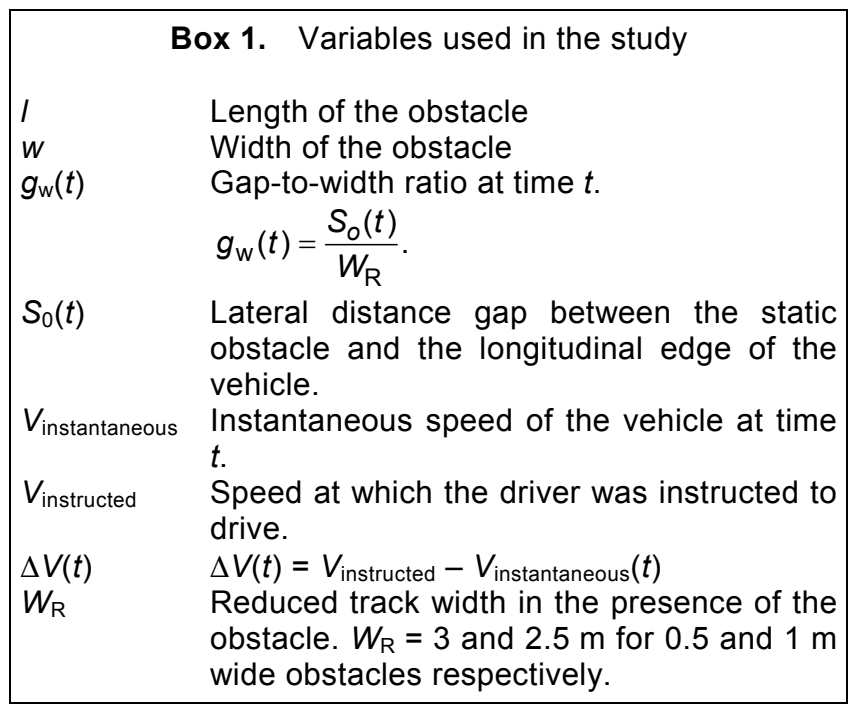


Table 1. One-tailed $t$-test on the influence of side of obstacle on gap-to-width ratio $g_{\mathrm{w}}(t)$

\begin{tabular}{|c|c|c|c|c|c|c|c|c|}
\hline \multirow[b]{2}{*}{$l(\mathrm{~m})$} & \multirow[b]{2}{*}{$V_{\text {instructed }}(\mathrm{m} / \mathrm{s})$} & \multirow[b]{2}{*}{$w(\mathrm{~m})$} & \multicolumn{2}{|c|}{$\begin{array}{l}g_{\mathrm{w}}(t) \text { when the obstacle is on } \\
\text { left hand side (LHS; m) }\end{array}$} & \multicolumn{2}{|c|}{$\begin{array}{l}g_{\mathrm{w}}(t) \text { when the obstacle is on } \\
\text { right hand side (RHS; m) }\end{array}$} & \multirow[b]{2}{*}{$t$-Value } & \multirow[b]{2}{*}{ Decision } \\
\hline & & & Mean & Std. dev & Mean & Std. dev & & \\
\hline \multirow[t]{4}{*}{25} & 8.4 & 0.5 & 0.35 & 0.07 & 0.28 & 0.02 & 6.45 & Reject $H_{0}$ \\
\hline & 11.2 & & 0.32 & 0.05 & 0.31 & 0.08 & 2.84 & Reject $H_{0}$ \\
\hline & 14 & & 0.28 & 0.08 & 0.25 & 0.08 & 1.78 & Do not reject $H_{0}$ \\
\hline & 14 & & 0.20 & 0.06 & 0.18 & 0.06 & 1.58 & Do not reject $H_{0}$ \\
\hline \multirow[t]{4}{*}{100} & 8.4 & 0.5 & 0.34 & 0.06 & 0.27 & 0.03 & 7 & Reject $H_{0}$ \\
\hline & 11.2 & & 0.33 & 0.05 & 0.28 & 0.06 & 4.29 & Reject $H_{0}$ \\
\hline & 14 & & 0.36 & 0.07 & 0.31 & 0.07 & 3.39 & Reject $H_{0}$ \\
\hline & 8.4 & 1 & 0.30 & 0.07 & 0.24 & 0.05 & 4.68 & Reject $H_{0}$ \\
\hline
\end{tabular}

obstacle is farther from him/her. Because of the diminished ability to perceive the lateral gap, the driver may maintain a higher $g_{\mathrm{w}}(t)$ when the obstacle is present on the passenger's side of the vehicle. To determine if this holds true, a one tailed $t$-test was performed to evaluate the influence of the location of the obstacle on $g_{\mathrm{w}}(t)$. The null and alternate hypotheses are provided in eq. (1). Table 1 shows the results of the $t$-test.

$H_{0}$ : Mean $g_{\mathrm{w}}(t)$ when the obstacle is on the left-hand side $\left(\right.$ LHS) of the vehicle $=$ mean $g_{\mathrm{w}}(t)$ when the obstacle is on RHS of the vehicle.

$H_{1}$ : Mean $g_{\mathrm{w}}(t)$ when the obstacle is on LHS of the vehicle $>$ mean $g_{\mathrm{w}}(t)$ when the obstacle is on RHS of the vehicle

At $95 \%$ confidence level, $H_{0}$ can be rejected in all but two observations, supporting the claim that drivers tend to maintain a higher lateral gap, $S_{0}(t)$, when the obstacle is laterally located farther from them.

The interception mechanisms adopted by drivers were to modify either the speed or the lateral gap.

\section{Influence of static obstacle width}

In this study, the two obstacles were 0.5 and $1 \mathrm{~m}$ wide.

Influence on gap-to-width ratio: Drivers tend to veer towards the centre of the road when its width decreases ${ }^{4,5}$, likely because the road edge poses a threat to safety ${ }^{4}$. As drivers position themselves laterally away from an obstacle, they also consider its position with respect to the available road width. It is expected that, in the presence of a wide obstacle, drivers will tend to offset the increased risk of being closer to the road edge by maintaining a lower $S_{0}(t)$ with the obstacle. That is, $g_{\mathrm{w}}(t)$ near the $1 \mathrm{~m}$ wide obstacle will be lower than that near the
$0.5 \mathrm{~m}$ wide obstacle. To validate this assumption, the hypotheses given in eq. (2) were tested with a one-tailed $t$-test. Table 2 shows the results of the $t$-test. At $95 \%$ confidence level, $H_{0}$ can be rejected in all but one observation, supporting this claim.

$H_{0}$ : Mean $g_{\mathrm{w}}(t)$ in the presence of $0.5 \mathrm{~m}$ wide obstacle $=$ mean $g_{\mathrm{w}}(t)$ in the presence of $1 \mathrm{~m}$ wide obstacle.

$H_{1}$ : Mean $g_{\mathrm{w}}(t)$ in the presence of $0.5 \mathrm{~m}$ wide obstacle $>$ mean $g_{\mathrm{w}}(t)$ in presence of $1 \mathrm{~m}$ wide obstacle.

Influence on deviation in instantaneous speed: A driver's manoeuvring space on the road decreases with increase in obstacle width. In order to safely manoeuvre around a wide obstacle, drivers might reduce the speed more than they would for a narrow obstacle. Hence, it was expected that $\Delta V(t)$ for a $1 \mathrm{~m}$ wide obstacle would be greater than that for a $0.5 \mathrm{~m}$ wide obstacle. To validate this assumption, the null and alternate hypotheses given in eq. (3) were tested with a one-tailed $t$-test. Table 3 shows the results. At $95 \%$ confidence level, $H_{0}$ could be rejected in all but one observation, which supports this claim.

$H_{0}$ : Mean $\Delta V(t)$ in the presence of $0.5 \mathrm{~m}$ wide obstacle $=$ mean $\Delta V(t)$ in the presence of $1 \mathrm{~m}$ wide obstacle.

$H_{1}$ : Mean $\Delta V(t)$ in the presence of $0.5 \mathrm{~m}$ wide obstacle $<$ mean $\Delta V(t)$ in the presence of $1 \mathrm{~m}$ wide obstacle.

\section{Influence of static obstacle length}

The perception of threat posed by an obstacle increases with increase in vehicle speed, size of the obstacle and duration of exposure. For the same width of obstacle, $g_{\mathrm{w}}(t)$ values for 25 and $100 \mathrm{~m}$ lengths were compared. 
Table 2. One-tailed $t$-test on the influence of width of obstacle on $g_{\mathrm{w}}(t)$

\begin{tabular}{|c|c|c|c|c|c|c|c|c|}
\hline \multirow[b]{2}{*}{$l(\mathrm{~m})$} & \multirow[b]{2}{*}{ Side of obstacle } & \multirow[b]{2}{*}{$V_{\text {instructed }}(\mathrm{m} / \mathrm{s})$} & \multicolumn{2}{|c|}{$g_{\mathrm{w}}(t)$ for $0.5 \mathrm{~m}$ wide obstacle } & \multicolumn{2}{|c|}{$g_{\mathrm{w}}(t)$ for $1 \mathrm{~m}$ wide obstacle } & \multirow[b]{2}{*}{$t$-Value } & \multirow[b]{2}{*}{ Decision } \\
\hline & & & Mean & Std. dev & Mean & Std. dev & & \\
\hline \multirow[t]{6}{*}{25} & LHS & 8.4 & 0.35 & 0.07 & 0.29 & 0.06 & 4.37 & Reject $H_{0}$ \\
\hline & & 11.2 & 0.32 & 0.05 & 0.22 & 0.06 & 8.59 & Reject $H_{0}$ \\
\hline & & 14 & 0.28 & 0.08 & 0.20 & 0.06 & 5.37 & Reject $H_{0}$ \\
\hline & RHS & 8.4 & 0.27 & 0.02 & 0.26 & 0.03 & 1.86 & Do not reject $H_{0}$ \\
\hline & & 11.2 & 0.31 & 0.08 & 0.20 & 0.04 & 8.25 & Reject $H_{0}$ \\
\hline & & 14 & 0.25 & 0.08 & 0.18 & 0.06 & 4.7 & Reject $H_{0}$ \\
\hline \multirow[t]{6}{*}{100} & LHS & 8.4 & 0.34 & 0.06 & 0.30 & 0.07 & 2.91 & Reject $H_{0}$ \\
\hline & & 11.2 & 0.33 & 0.05 & 0.26 & 0.10 & 4.2 & Reject $H_{0}$ \\
\hline & & 14 & 0.36 & 0.07 & 0.29 & 0.07 & 4.74 & Reject $H_{0}$ \\
\hline & RHS & 8.4 & 0.27 & 0.03 & 0.24 & 0.05 & 3.45 & Reject $H_{0}$ \\
\hline & & 11.2 & 0.28 & 0.06 & 0.22 & 0.08 & 4.02 & Reject $H_{0}$ \\
\hline & & 14 & 0.29 & 0.07 & 0.24 & 0.08 & 3.16 & Reject $H_{0}$ \\
\hline
\end{tabular}

Table 3. One tailed $t$-test on the influence of width of obstacle on deviation in instantaneous speed $\Delta V(t)$

\begin{tabular}{|c|c|c|c|c|c|c|c|c|}
\hline \multirow[b]{2}{*}{$l(\mathrm{~m})$} & \multirow[b]{2}{*}{ Side of obstacle } & \multirow[b]{2}{*}{$V_{\text {instructed }}(\mathrm{m} / \mathrm{s})$} & \multicolumn{2}{|c|}{$\Delta V(t)$ for $0.5 \mathrm{~m}$ wide obstacle } & \multicolumn{2}{|c|}{$\Delta V(t)$ for $1 \mathrm{~m}$ wide obstacle } & \multirow[b]{2}{*}{$t$-Value } & \multirow[b]{2}{*}{ Decision } \\
\hline & & & Mean & Std. dev & Mean & Std. dev & & \\
\hline \multirow[t]{6}{*}{25} & LHS & 8.4 & 0.29 & 0.51 & 0.59 & 0.83 & -2.07 & Reject $H_{0}$ \\
\hline & & 11.2 & 0.18 & 0.49 & 0.54 & 0.89 & -2.38 & Reject $H_{0}$ \\
\hline & & 14 & 0.8 & 0.43 & 1.36 & 0.46 & -5.97 & Reject $H_{0}$ \\
\hline & RHS & 8.4 & -0.05 & 0.73 & 0.74 & 1.11 & -3.99 & Reject $H_{0}$ \\
\hline & & 11.2 & 0.58 & 0.28 & -0.06 & 0.59 & 6.57 & Reject $H_{0}$ \\
\hline & & 14 & 0.66 & 0.42 & 0.94 & 0.57 & -2.65 & Reject $H_{0}$ \\
\hline \multirow[t]{6}{*}{100} & LHS & 8.4 & 0.06 & 0.28 & 0.93 & 1.52 & -3.78 & Reject $H_{0}$ \\
\hline & & 11.2 & 0.12 & 0.5 & 0.99 & 2.05 & -2.77 & Reject $H_{0}$ \\
\hline & & 14 & 1.5 & 2.61 & 2.57 & 3.28 & -2.11 & Reject $H_{0}$ \\
\hline & RHS & 8.4 & 0.43 & 0.89 & 0.51 & 0.62 & -0.49 & Do not reject $H_{0}$ \\
\hline & & 11.2 & 1.17 & 1.98 & 1.48 & 1.89 & -0.76 & Do not reject $H_{0}$ \\
\hline & & 14 & 1.29 & 2.13 & 3.31 & 3.31 & -3.44 & Reject $H_{0}$ \\
\hline
\end{tabular}

Similarly, $\Delta V(t)$ values for 25 and $100 \mathrm{~m}$ lengths were compared.

Influence on gap-to-width ratio: The null and alternate hypotheses related to the influence of obstacle length are given in eq. (4). These were tested with a one-tailed $t$-test. It is intuitive that an increase in exposure time with a larger obstacle causes higher threat perceptions. Given that the duration of exposure with a $100 \mathrm{~m}$ long obstacle is greater than with $25 \mathrm{~m}$ long obstacle, $g_{\mathrm{w}}(t)$ should be greater for the $100 \mathrm{~m}$ long obstacle and hence $H_{1}$ was chosen. Table 4 shows the results of the $t$-test.

$H_{0}$ : Mean $g_{\mathrm{w}}(t)$ in the presence of $25 \mathrm{~m}$ long obstacle $=$ mean $g_{\mathrm{w}}(t)$ in the presence of $100 \mathrm{~m}$ long obstacle.

$H_{1}$ : Mean $g_{\mathrm{w}}(t)$ in the presence of $25 \mathrm{~m}$ long obstacle $<$ mean $g_{\mathrm{w}}(t)$ in the presence of $100 \mathrm{~m}$ long obstacle.

An interesting observation can be made from Table 4. At $95 \%$ confidence level, $H_{0}$ is always rejected when $V_{\text {instructed }}$ is $14 \mathrm{~m} / \mathrm{s}$, indicating that obstacle length influences $g_{w}(t)$ only at higher driving speed. This is evident because the perceived risk in the presence of an obstacle increases with increase in vehicle speed. Hence, it can be inferred that at higher speeds, drivers prefer to position themselves laterally farther away from a longer obstacle compared to a shorter obstacle of the same width. Again, $H_{0}$ is rejected when the $1 \mathrm{~m}$ wide obstacle is present on the LHS of the vehicle and $V_{\text {instructed }}$ is $14 \mathrm{~m} / \mathrm{s}$. This might be attributed to greater width of the obstacle, because such behaviour was not observed when the obstacle was $0.5 \mathrm{~m}$ wide.

Influence on deviation in instantaneous speed: Larger obstacles result in higher levels of perceived risk ${ }^{1,20}$. Hence, it was expected that $\Delta V(t)$ would be greater for the $100 \mathrm{~m}$ obstacle than for the $25 \mathrm{~m}$ obstacle. The hypotheses shown in eq. (5) reflect this assumption, and they were tested with a one-tailed $t$-test. Table 5 shows the results. At $95 \%$ confidence level, $H_{0}$ can be rejected in all but two observations, which supports this claim.

$H_{0}$ : Mean $\Delta V(t)$ in the presence of $25 \mathrm{~m}$ long obstacle $=$ mean $\Delta V(t)$ in the presence of $100 \mathrm{~m}$ long obstacle.

$H_{1}$ : Mean $\Delta V(t)$ in the presence of $25 \mathrm{~m}$ long obstacle $<$ mean $\Delta V(t)$ in the presence of $100 \mathrm{~m}$ long obstacle. 
Table 4. One-tailed $t$-test on the influence of length of obstacle on $g_{\mathrm{w}}(t)$

\begin{tabular}{|c|c|c|c|c|c|c|c|c|}
\hline \multirow[b]{2}{*}{$w(\mathrm{~m})$} & \multirow[b]{2}{*}{ Side of obstacle } & \multirow[b]{2}{*}{$V_{\text {instructed }}(\mathrm{m} / \mathrm{s})$} & \multicolumn{2}{|c|}{$g_{\mathrm{w}}(t)$ for $25 \mathrm{~m}$ long obstacle } & \multicolumn{2}{|c|}{$g_{\mathrm{w}}(t)$ for $100 \mathrm{~m}$ long obstacle } & \multirow[b]{2}{*}{$t$-Value } & \multirow[b]{2}{*}{ Decision } \\
\hline & & & Mean & Std. dev & Mean & Std. dev & & \\
\hline \multirow[t]{6}{*}{0.5} & LHS & 8.4 & 0.35 & 0.07 & 0.34 & 0.06 & 0.73 & Do not reject $H_{0}$ \\
\hline & & 11.2 & 0.32 & 0.05 & 0.33 & 0.05 & -0.95 & Do not reject $H_{0}$ \\
\hline & & 14 & 0.28 & 0.08 & 0.36 & 0.07 & -5.05 & Reject $H_{0}$ \\
\hline & RHS & 8.4 & 0.28 & 0.02 & 0.27 & 0.03 & 1.86 & Do not reject $H_{0}$ \\
\hline & & 11.2 & 0.31 & 0.08 & 0.28 & 0.06 & 2.01 & Reject $H_{0}$ \\
\hline & & 14 & 0.25 & 0.08 & 0.31 & 0.07 & -3.79 & Reject $H_{0}$ \\
\hline \multirow[t]{6}{*}{1} & LHS & 8.4 & 0.29 & 0.06 & 0.30 & 0.07 & -0.73 & Do not reject $H_{0}$ \\
\hline & & 11.2 & 0.22 & 0.06 & 0.26 & 0.1 & -2.3 & Reject $H_{0}$ \\
\hline & & 14 & 0.2 & 0.06 & 0.29 & 0.07 & -6.55 & Reject $H_{0}$ \\
\hline & RHS & 8.4 & 0.25 & 0.03 & 0.24 & 0.05 & 1.15 & Do not reject $H_{0}$ \\
\hline & & 11.2 & 0.21 & 0.04 & 0.22 & 0.08 & -0.75 & Do not reject $H_{0}$ \\
\hline & & 14 & 0.18 & 0.06 & 0.24 & 0.08 & -4.02 & Reject $H_{0}$ \\
\hline
\end{tabular}

Table 5. One-tailed $t$-test on the influence of length of obstacle on $\Delta V(t)$

\begin{tabular}{|c|c|c|c|c|c|c|c|c|}
\hline \multirow[b]{2}{*}{$w(\mathrm{~m})$} & \multirow[b]{2}{*}{ Side of obstacle } & \multirow[b]{2}{*}{$V_{\text {instructed }}(\mathrm{m} / \mathrm{s})$} & \multicolumn{2}{|c|}{$\Delta V(t)$ for $25 \mathrm{~m}$ long obstacle } & \multicolumn{2}{|c|}{$\Delta V(t)$ for $100 \mathrm{~m}$ long obstacle } & \multirow[b]{2}{*}{$t$-Value } & \multirow[b]{2}{*}{ Decision } \\
\hline & & & Mean & Std. dev & Mean & Std. dev & & \\
\hline \multirow[t]{6}{*}{0.5} & LHS & 8.4 & 0.29 & 0.51 & 0.06 & 0.28 & 2.8 & Reject $H_{0}$ \\
\hline & & 11.2 & 0.18 & 0.49 & 0.13 & 0.5 & 0.51 & Do not reject $H_{0}$ \\
\hline & & 14 & 0.80 & 0.43 & 1.50 & 2.61 & -2.07 & Reject $H_{0}$ \\
\hline & RHS & 8.4 & -0.05 & 0.73 & 0.43 & 0.89 & -2.95 & Reject $H_{0}$ \\
\hline & & 11.2 & 0.58 & 0.28 & 1.17 & 1.98 & -2.09 & Reject $H_{0}$ \\
\hline & & 14 & 0.66 & 0.42 & 1.29 & 2.13 & -2.05 & Reject $H_{0}$ \\
\hline \multirow[t]{6}{*}{1} & LHS & 8.4 & 0.59 & 0.83 & 1.04 & 1.52 & -1.96 & Reject $H_{0}$ \\
\hline & & 11.2 & 0.55 & 0.89 & 0.99 & 2.05 & -1.39 & Do not reject $H_{0}$ \\
\hline & & 14 & 1.35 & 0.46 & 2.57 & 3.28 & -2.6 & Reject $H_{0}$ \\
\hline & RHS & 8.4 & 0.74 & 1.11 & 0.51 & 0.62 & 1.28 & Do not reject $H_{0}$ \\
\hline & & 11.2 & -0.06 & 0.59 & 1.49 & 1.89 & -5.54 & Reject $H_{0}$ \\
\hline & & 14 & 0.94 & 0.57 & 3.31 & 3.31 & -4.99 & Reject $H_{0}$ \\
\hline
\end{tabular}

\section{Conclusion}

Few studies have examined the influence of the position of a static obstacle (driver's side versus passenger's side) and its dimensions (width and length) on lateral gap and speed. The objective of the present study was to gain further understanding of the influence of on-road static obstacles on driver behaviour. An experiment was conducted using an instrumented vehicle on a test track in which the static obstacles caused a reduction in the track width.

The finding indicates that driver behaviour is influenced by the side on which the obstacle is present; drivers prefer to maintain a larger lateral gap when the obstacle is located on the passenger's side. This behaviour could be attributed to the fact that the driver's ability to judge the lateral gap becomes poorer when he/she is laterally farther away from the obstacle, i.e. when the obstacle is located on the passenger side of the vehicle. The impact of the influence of the side of the obstacle can be used to study driver response in different instances, such as when a vehicle approaches a left-hand and right-hand road turnings, and when it approaches pedestrians and other vehicles on the road. The findings from these studies can be used in lateral control models of microscopic traffic flow.

Most of the existing literature discusses the influence of overall size of the obstacle. However, the influence of individual dimensions of on-road obstacles has not been studied. From the present study, it can be inferred that both obstacle width and length influence vehicle speed and the lateral gap. However, the influence of obstacle length on the lateral gap is prominent only at higher vehicle speed. The impact of different dimensions can be used to study the size-arrival effect while determining collision potential in traffic safety analysis.

In this study we evaluate the influence of width and length of an on-road static obstacle individually. However, only two different widths, two different lengths and three different speeds are considered. In order to get qualitative insights, it is important that other combinations of widths, lengths and speeds should also be considered. The original track width is $3.5 \mathrm{~m}$. The track width can be varied gradually in order to evaluate the track width at which the influence of obstacle vanishes. The influence of height of obstacle can also be studied. Besides, the number of 


\section{RESEARCH ARTICLES}

drivers can be increased in order to collect data from a larger pool of drivers. This study was conducted with only five drivers. In order to get a better insight, the analysis can be done with more number of drivers.

1. DeLucia, P. R. and Warren, R., Pictorial and motion-based depth information during active control of self-motion: size-arrival effects on collision avoidance. J. Exp. Psychol.: Hum. Percept. Perform., 1994, 20(4), 783-798.

2. DeLucia, P. R., Effects of size on collision perception and implications for perceptual theory and transportation safety. Psychol. Sci., 2013, 22(3), 199-204.

3. Monograph on Traffic Flow Theory: A State-of-the-Art, 2001.

4. Chakroborty, P., Agrawal, S. and Vasishtha, K., Microscopic modeling of driver behaviour in uninterrupted traffic flow. J. Transport. Eng., 2004, 130(4), 438-451.

5. Rosey, F., Auberlet, J. M., Moisan, O. and Dupré, G., Impact of narrower lane width comparison between fixed-base simulator and real data. Transp. Res. Rec.: J. Transp. Res. Board, 2009, 2138, 112-119.

6. Yousif, S., Nassrullah, Z. and Norgate, S. H., Narrow lanes and their effect on drivers' behaviour at motorway roadworks. Transport. Res. Part F, 2017, 47, 86-100.

7. Green, P., Lin, B. and Bagian, T., Driver workload as a function of road geometry: a pilot experiment. Report No. GLCTTR 22-91/01, Transportation Research Institute, Ann Arbor, Mich, USA, 1994.

8. Bassat, T. B. and Shinar, D., Effect of shoulder width, guardrail and roadway geometry on driver perception and behaviour. Accid. Anal. Prev., 2011, 43, 2142-2152.

9. De Waard D., Jessurun, M., Steyvers, F. J., Raggatt, P. T. and Brookhuis, K. A., Effect of road layout and road environment on driving performance, drivers' physiology and road appreciation. Ergonomics, 1955, 38(7), 1395-1407.

10. Marten, M., Comte, S. and Kaptein, N., The effects of road design on speed behaviour: a literature review, Deliverable D1 (Report 2.3.1), MASTER, 1997.
11. Godley, S. T., Triggs, T. J. and Fildes, B. N., Perceptual lanewidth, wide perceptual road centre marking and driving speeds. Ergonomics, 2004, 47(3), 237-256.

12. Shinar, D., In Traffic Safety and Human Behaviour, Elsevier, Oxford, UK, 2000.

13. Stamatiadis, N., Bailey, K., Grossardt, T. and Ripy, J., Evaluation of highway design parameters on influencing operator speeds through case wise visual evaluation. Transp. Res. Rec.: J. Transp. Res. Board, 2010, 219, 143-149.

14. Michaels, R. M. and Cozan, L. W., Perceptual and field factors causing lateral displacement. Highway Res. Rec., 1963, 25, 1-13.

15. Taragin, A., Driver behaviour as affected by static objects on highway shoulders. Highway Transport. Res. Boards, Bureau of Public Roads, 1955, 1445, 34-46.

16. Van Der Horst, R. and De Ridder, S., Influence of roadside infrastructure on driving behaviour: driving simulator study. J. Exp. Phychol. Learn: Hum. Percept. Perform., 2007, 2018(1), 36-44.

17. Martin, P. L., Audet, T., Corriveau, H., Hamel, M., D’Amours, M. and Smeesters, C., Comparison between younger and older drivers of the effect of obstacle direction on the minimum obstacle distance to brake and avoid a motor vehicle accident. Accid. Anal. Prev., 2010, 42, 1144-1150.

18. DeLucia, P. R., Pictorial and motion-based information for depth perception. J. Exp. Phychol. Learn: Hum. Percept. Perform., 1991, 17, 738-748.

19. DeLucia, P. R., Does binocular disparity or familiar size override effects of relative size on judgments of time to contact. J. Exp. Psychol., 2005, 58A, 865-886.

20. Caird, J. K. and Hancock, P. A., The perception of arrival time for different oncoming vehicles at an intersection. Ecol. Psychol., 1994, 6, 83-109.

21. Levulis, S. J., DeLucia, P. R. and Jupe, J., Effects of oncoming vehicle size on overtaking judgments. Accid. Anal. Prev., 2015, 82, $163-170$.

Received 6 June 2020; revised accepted 5 November 2020

doi: $10.18520 / \mathrm{cs} / \mathrm{v} 120 / \mathrm{i} 4 / 699-706$ 